\title{
NEW WAY OF MONITORING OF THE PRODUCTION ENVIRONMENT WITH APPLICATION OF AUGMENTED REALITY AND ARTIFICIAL INTELLIGENCE
}

doi:10.2478/mape-2018-0039

Date of submission of the article to the Editor: 04/2018

Date of acceptance of the article by the Editor: 07/2018
MAPE 2018, volume 1, issue 1, pp. 307-313

\author{
Andrzej Szajna \\ Digital Technology Poland (DTP Ltd.), Poland \\ Prof. Janusz Szajna \\ Digital Technology Poland (DTP Ltd.), Poland \\ University of Zielona Góra, Poland \\ Dr hab. inż. Roman Stryjski, prof. UZ \\ University of Zielona Góra, Poland \\ Dr. hab. inż. Josef Bas। \\ University of Zielona Góra, Poland \\ University of West Bohemia, Czech Republic \\ Dr hab. inż. Jarosław Brodny \\ Silesian University of Technology, Poland
}

\begin{abstract}
Augmented reality technology (AR), also known as mixed reality, allows to enhance the seen reality with additional, virtual objects, displayed right in front of the user's eyes. The article presents the concept of using Augmented Reality glasses, to support the user with pre-defined knowledge and instructions, giving the ability to do the job without almost any idea of what should be done. The operator simply follows the instructions shown in front of the eyes and communicate with the system with simple gesture and voice. Additionally, the article touches the topic of Artificial Intelligence (Al) which will allow the system in the future to have not only the pre-defined, hard coded instructions, but also the flexibility to give different hints to the user based on a different situation observed.
\end{abstract}

Keywords: Augmented Reality, monitoring and inspection of the factory, AR glasses

\section{INTRODUCTION}

With the digital revolution and the fourth industrial revolution, we are facing waves of technology that will dominate investment and innovation for at least a decade (Brodny et al., 2016a; Brodny et al., 2016b; Brodny et al., 2017a; Brodny et al., 2017b; Burdea and Coiffet, 2003; Meni, 2006; Stecuła et al. 2018). New possibilities arise with the combination of advanced ICT engineering, Augmented Reality (AR) and Artificial Intelligence (AI) (Haller, 2006; Yu et al., 2010; Zhou, 2008). The AR revolution, that is strongly seen in the last 3 years, when new glasses were introduced by world's biggest companies, opened new flexible and natural possibilities of the human-machine communication and interaction. The leading industry companies shows increasing research in this topic with real life applications that can be observed during the biggest trade fairs.

The Gartner Group estimates that $\$ 1200$ billion will be invested in Al systems and services this year (Zhou et al., 2008). This already applies to the entire value chain around artificial intelligence. Wherever Al systems are used, revenue leaps through predictive analytics, 
communication benefits through voice control, efficiencies through automation, insight through cognitive computing, and increased flexibility through machine learning and deep learning. There is a race to find new algorithms and ways of solving problems in these fields, making huge demand for advanced, scientific research and development.

\section{THE DIGITAL REVOLUTION IN INDUSTRY AND AUGMENTED REALITY AS THE ANSWER TO NATURAL HUMAN-MACHINE INTERACTION NEED IN THE INSPECTION AND MONITORING OF PRODUCTION PROCESSES}

The industry faces the big change. There already is a high level of automation but on the other hand, there is significant amount of places where digitalization is highly needed: paperwork and printed PDFs, transferring files on a pen-drive, unrecognizable formats through different machines and systems are only few examples. Additionally, big concerns about security and data protection, result in slowdown or even unwillingness for digital change. However, to constantly improve processes, allow for user customization of a product and shorten the delivery time, digitalization, standardization and implementation of artificial intelligence is the only possible way.One of the key elements in digitalization is to strive to create an easy to use, flexible and natural human-machine interface. Augmented Reality (AR) (Łunarski, 2000) seems to give such solution. Advanced DTPoland-UZ works show that AR with proprietary software and connection to cloud provides a very useful and powerful tool for inspection and monitoring of a factory. Issues of uninterrupted production process, proper functioning and effective diagnostics are now supported with intuitive and user friendly system. The pre-defined instructions, connected to the flexibly judged, based on real-time data from sensors and meters, current situation of eg. machine behaviour, enhanced with hints and alerts, allow a user to do the job about $20-30 \%$ faster with reports created instantly, visible to managers in real-time. All the data is gathered in the cloud, processed and directed to obtain noticeable trends and future valuable hints. This helps to constantly improve the system and even better support the operator. Solutions of this type allow the life-span of individual parts to be utilised to their fullest extent by optimising their replacement dates and controlling their operating processes; this, then translates into measurable financial savings and impacts, ultimately, on the financial condition of an enterprise.

One of the most interesting and flexible for the user options of the Augmented Reality tool are the projection glasses - in simplified terms, two miniaturised projectors mounted over each glass in a casing thus allowing them to be worn in front of the eyes in a manner analogous to traditional, eyeglasses or sunglasses.

\section{STRUCTURE AND OPERATING PRINCIPLES OF A SYSTEM USING AUG-MENTED REALITY TECHNOLOGY}

As part of the R\&D work carried out at the Digital Technology Poland (DTP Ltd.) in Zielona Góra and at the Institute of Computer Science and Production Management at the University of Zielona Góra (UZ), systems using Augmented Reality Technology to support technical personnel in the inspection and monitoring of production lines, have been developed. By implementing the functionality in the form of interchangeable instructions, delivered in online mode, it is possible for the system operator to carry out inspection work with neither training nor with the help of an instructor.

The main elements of the structure of the system developed are: edge computing devices connected to each of the machines or group of machines, sensors and meters (including energy meters), cloud resources for different data tasks and preparation on visualization, AR glasses (Cakmakci and Rolland, 2006) proprietary DTPoland-UZ image processing and analysis software and, optionally, a keypad. The AR glasses, depending on the requirements of the system operator, may be ex-changed with a tablet. The system works in real time. Virtual elements are generated by proprietary DTPoland-UZ software on the basis of 
prepared logic, digital instructions and real-time data from AR glasses cameras and measuring devices. The image displayed by the projection device is enriched with virtual objects in the form of 3D models (Dugelay, 2008), virtual screens (dashboards) and virtual buttons. The operator sees the exact instructions, locations and activities necessary to perform the assembly or inspection work. The positions of virtual objects are calculated relative to pre-located virtual markers (Sun et al., 2011; Uchiyama and Marchan, 2012), placed right next to the inspected machine. In the future, markers will be replaced with advanced image recognition techniques that apply Artificial Intelligence (Al), that is, deep convolutional neural networks, which the DTPoland-UZ team are currently working on.

The algorithm of real image enrichment with virtual objects is as follows:

- recognition of virtual markers placed near the inspected machine,

- connection to the cloud for receiving the corresponding data for this concrete machine and its state,

- recognition of the machine based on the pre-mapped surrounding,

- calculation of the co-ordinates of virtual objects relative to reference systems and the orientation of virtual markers and the machine,

- rendering the virtual objects and displaying them on the AR glasses, on the observed by system operator real environment.

The result of the algorithm is the seen image of the machine enriched with virtual objects (Fig. 1).

The system's software consists of an application programme for synchronising the exact place where the operator stays with cloud data - status of the monitored machine, along with the set of instructions dedicated to the selected inspection processes.

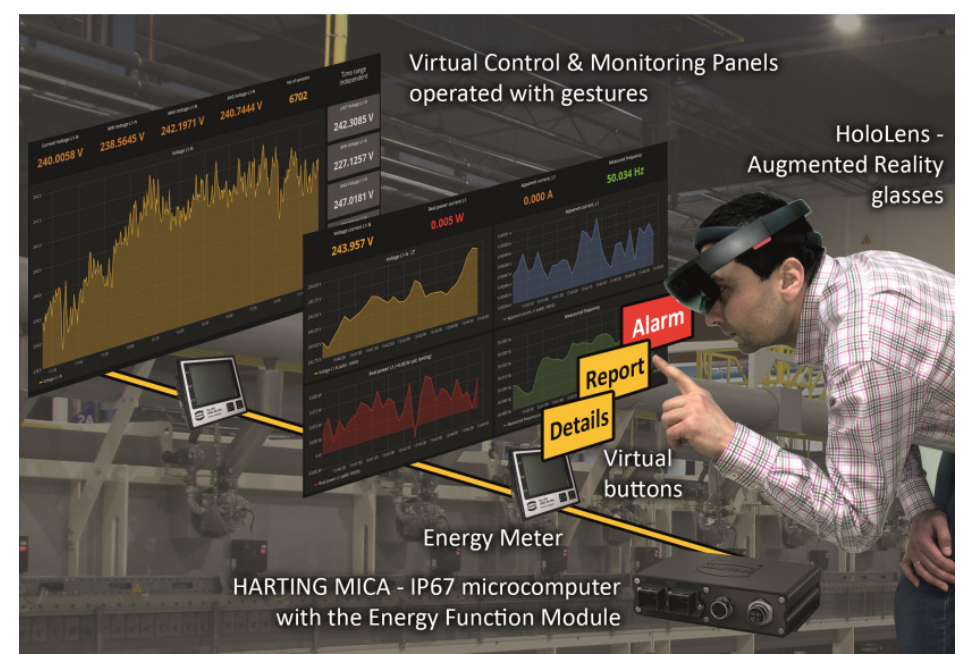

Fig. 1. Virtual objects, such as, displays, dashboards, buttons or suggested path to follow (symbolized with the yellow line), embedded on the actual observed scene - a production line Source: (Business Way, 2018).

\section{METHOD FOR SUPPORTING THE INSPECTION AND MONITORING PROCESS}

Supporting the inspection and monitoring process with the use of the system developed, is achieved through a visual presentation to the operator: virtual markers that show that the machine behind can be inspected, descriptive prompts in textual form, measurement values, status information and alerts, 3D models of the machine's components (eg. for potential replacement).

The methodology also assumes application of the system in such areas as:

- recognition of the technical condition of the device, that is, technical diagnostics and identification of the causes of possible failures, 
- repairs (i.e. activities aimed at restoring the functional properties of the device that have been damaged, during which the regeneration or replacement of the damaged components are carried out),

- maintenance operations, that is, activities recommended by the manufacturer of the technical device in order to maintain the components of the device in such a condition as to allow trouble-free operation,

- training of technical staff, by improving their skills and by their acquisition of the qualifications necessary to undertake works related to the assembly, recognition of technical condition, repair and maintenance of the technical device.

Visualisation is carried out through AR glasses - a part of the user's communication interface with the system. It allows the operator to have full freedom of movement during the work, since the glasses do not engage his/her hands or affect his/her mobility.

The user's interaction with the system takes place through simple gestures or speech. The optional keypad allows the user an alternative way to move around after each point of the programme or the instructions prepared. Individual points in the instructions can be attended to without feedback regarding the course of the inspection process or requiring return signals. The instructional data synchronisation programme, located in the cloud part of the system, is used to collect feedback. The instruction data synchronisation programme reads information about the status of the monitored machine and the inspection process, based on the data provided by the software of the measuring system and inputs an appropriate entry into the database. This entry is read by an instruction programme that displays information supporting the operator, in such as, information about the correctness of the activity being performed; this allows the user to go to the next point in the instruction set. In the method developed with the application of augmented reality, the important issue is the interaction between the content of the instructions, enriched with virtual objects, and the user, as well as the physical machine.

The physical and logical elements involved in the inspection and training processes influence the decisions made by the operator. The operator, based on the seen machine, enriched with the content of the virtual instructions, interprets the status of the machine and point of inspection process and then decides on further action, as described in the instructions. The operator's actions, through feedback, in the course of the inspection process, are visually signalled, which, in turn, checks them in order to assess whether they have been carried out correctly. Based on additional visual inspection, the operator can add new comments to the machine's inspectin history by clicking virtual buttons, writing text notes or dictating a note with voice.

\section{AN EXAMPLE OF THE USE OF AUGMENTED REALITY TECHNOLOGY IN THE INSPECTION AND MONITORING OF THE PRODUCTION LINE}

Below is presented an example of the application of Augmented Reality in industry. It is the monitoring of the production line through, inter alia, measurements of energy consumption by particular parts of the technological sequence. This is done using the DTPoland AR Energy 4.0 system, consisting of a edge computing unite - HARTING MICA Energy microcomputer, Microsoft AZURE Cloud and visualisation on the Microsoft HoloLens Augmented Reality glasses (Powering the Industry 4.0..., 2017) (Fig. 2). 




Fig. 2. Diagram, monitoring the production line of power cabinets using Augmented Reality Technology

Monitoring a production line is a daily and necessary task in every factory. In modern, 4.0 factories, this is done with the use of advanced systems for automatic monitoring and warning and more and more often, also intelligent response. Usually, however, daily inspection is performed by a human being - a qualified factory worker. In this case, it is necessary to provide him/her with IT systems providing the best possible support, with transparent human/machine interfaces. Tablet devices are a great improvement for the employee performing the review, however they are still sub-optimal and cumbersome. In such a situation, one modern solution could be Augmented Reality glasses, which display to the user all the necessary data in front of his/her eyes, while all control, the making of notes and the writing of reports are done using gestures and speech.

The DTPoland AR Energy 4.0 system for visualising the parameters of electricity, consumed by robots or machines in a factory, is an example of the very user-friendly monitoring of a production line in a 4.0 factory using Augmented Reality glasses.

The system allows virtual screens or dashboards, to be positioned with graphs showing the quality and power consumption, directly above/next to/at robots/machines, without the need to mount additional visualisation devices at each workstation.

The system consists of three functional parts:

a) A HARTING MICA micro-computer with electricity meters for monitoring the robots connected to it;

b) Azure loT Hub Clouds with a database and data visualisation application;

c) Microsoft HoloLens Augmented Reality glasses.

The following assumptions were made in relation to the hardware of the HARTING MICA micro-computer:

- electricity meters use the MODBUS protocol,

- the meters are connected to the RS485 serial bus,

- the maximum number of meters that can be connected to the MICA microcomputer is 247 , 
- the RS485 serial bus, on the MICA micro-computer, is realised by an extension module, designed by the DTPoland and enabling the data to be written and read directly from the operating system,

- in addition, the MICA expansion module (FM board) creates 8 virtual MODBUS devices, corresponding to 8 inputs for the SO pulse energy meters.

HARTING MICA micro-computer software architecture, is based on the Linux system, in which functional modules are run using the LXC virtualisation method as separate microservices, viz., the operating system-level-level virtualisation method for running multiple systems isolated as Linux systems.

The DTPoland AR Energy 4.0 system uses three micro-services on the HARTING MICA micro-computer:

i. Data Collector - software that downloads data from electricity meters, in accordance with the configuration set and sends the data to the next container via the MQTT protocol;

ii. MQTT broker - the software used to transfer any data between devices and the broadcasting software, or the publisher and the receiving software, viz., the subscriber;

iii. Azure connector - the software which receives data from the MQTT broker and transmits it to the Azure loT Hub Cloud;

Data sent between containers is appropriately formatted, using JSON standard information about the measured values provided by the meters;

Azure loT Hub cloud:

With regard to Azure, the system consists of two components:

i. a database storing the measured values, each database record, or row, is time stamped;

ii. visualisation software which downloads data to charts from a database and displays dashboards in the form of an HTML application

Microsoft HoloLens Augmented Reality glasses allow visualisation of the virtual screens, or dashboards, to be controlled by gestures. The software created for the Microsoft HoloLens glasses, based on the Unity 3D product, that is, an engine for creating games and 3D applications using $\mathrm{C \#}$ programming language, allows communication with the system for visualisation to be installed in Azure Cloud. Thanks to the advanced techniques used to recognise spatial location and image recognition, the system combines real elements, such as factory robots with data for presentations downloaded from the Azure Cloud and displays them next to these real devices. The system also allows current, or archival, parameters to be presented, as also the characteristics of particular values, measured in the form of diagrams. Additionally, it is possible to view registered alarm statuses, their configuration as well as the configuration of the entire device.

\section{CONCLUSION}

The solution presented in this article, covering the methodology, data collection and processing in the cloud and visualization of the status of monitored machine, using the technology of augmented reality, is a significant step towards improving human/machine interaction and time needed to perform the job. Additionally, the system operator will be able to perform specific and advanced tasks without a need of training or any help of an qualified instructor. Thanks to the solution proposed, it is possible to (a) smoother move in the factory facility without any knowledge of its layout, (b) shorten inspection time, (c) reduce costs related to technical staff training, (d) reduce staff by broadening the scope of work performed by a single person and (e) support training for new employees. The use of this system, developed in industrial conditions, can have a significant impact on ensuring the continuity of production by reducing the risks associated with shortages of personnel responsible for maintenance, particularly in plants employing a small number of people and producing a wide range of products. 
The solution developed is open, which means that the system and the method proposed can be supplemented with new elements (eg. sensors) and instructional data, as well as the possibility of using the solution in other areas of application, such as the ongoing maintenance of technical devices and their repair.

\section{ACKNOWLEDGEMENTS}

The authors would like to thank the DTPoland engineering team, engaged in the project: Prof. Krzysztof Diks, Dr. Tomasz Kozlowski and also Marek Adaszyński, Piotr Charyna, Filip Chmielewski, Krzysztof Ciebiera, Joanna Cieniuch, Grzegorz Gajdzis, Anna Mroczkowska, Marcin Mucha, Sebastian Pawlak, Wojciech Regeńczuk, Eugeniusz Tswigun, Andrzej Warycha, Czesław Zubowicz, Mariusz Życiak

\section{REFERENCES}

Brodny J., Stecula K., Tutak M. (2016a). Application of the TPM strategy to analyze the effectiveness of using a set of mining machines. SGEM2016 Conference Proceedings, Book1 Vol. 2, 65-72 pp. DOI: 10.5593/SGEM2016/B12/S03.009.

Brodny J., Stecula K., Tutak M. (2016b). Application of the TPM strategy to analyze the effec-tiveness of using a set of mining machines. SGEM2016 Conference Proceedings, Book1 Vol. 2, 65-72 pp. DOI: 10.5593/SGEM2016/B12/S03.009.

Brodny J., Tutak M., Michalak M. (2017a): A Data Warehouse as an Indispensable Tool to Determine the Effectiveness of the Use of the Longwall Shearer. Beyond Databases, Architectures and Structures. Towards Efficient Solutions for Data Analysis and Knowledge Representation. BDAS 2017. 13th International Conference, BDAS 2017 Communications in Computer and Information Science, vol 716. Springer, Cham. pp 453-465. DOI: 10.1007/978-3-319-58274-0_36.

Brodny J., Tutak M., Michalak M. (2017b). The Use of the TGŚP Module as a Database to Identify Breaks in the Work of Mining Machinery. Beyond Databases, Architectures and Structures. Towards Efficient Solutions for Data Analysis and Knowledge Representation. BDAS 2017. 13th International Conference, BDAS 2017, Communications in Computer and Information Science, vol 716. Springer, Cham. pp 441-452. DOI: 10.1007/978-3-319-58274-0_35.

Burdea G. C., Coiffet P. (2003). Virtual Reality Technology. Second Edition, Wiley-IEEE Press..

Cakmakci O., Rolland J. (2006). Head-Worn Displays. Journal of Display Technology: A Review vol. 2.

Dugelay J. L., Baskurt A., Daoudi M. (2008). 3D Object Processing, Compression, Indexing and Watermarking. Wiley.

Haller M. (2006). Emerging Technologies of Augmented Reality. Idea Group Publishing, Hershey USA.

Łunarski J. (2000). O problemach elastycznego montażu. Tech. i Autom. Mont. nr 4.

Meni E. (2006). Boeing's working on augmented reality which could change space training ops. Boeing Frontiers vol. 10.

Powering the industry 4.0 revolution in manufacturing with Windows 10 and Microsoft Cloud (2017). Skand Mittal/Windows Industry Lead.

Stecuła K., Brodny J., Tutak M. (2018). Use of intelligent informatics module for registration and assessment of causes of breaks in selected mining machines. Intelligent Systems in Production Engineering and Maintenance ISPEM 2017, Advances in Intelligent Systems and Computing 637, pp.74-84.DOI 10.1007/978-3-319-64465-3_8

Sun R., Sui Y., Li R., Shao F.: The Design of a New Marker in Augmented Reality. IPEDR vol. 4. IACSIT Press, Singapore 2011.

Uchiyama H., Marchand E. (2012).Object detection and pose tracking for augmented reality: Recent approaches. 18th Korea-Japan Joint Workshop on Frontiers of Computer Vision (FCV).

Yu D., Sheng Jin J., Luo S., Lai W., Huang Q. (2010). A Useful Visualization Technique: A Literature Review for Augmented Reality and its Application, limitation \& future direction. Visual Information Communication. Springer.

Zhou F., Duh H. B. L., Billinghurst M. (2008). Trends in augmented reality tracking, interaction and display: A review of ten years of ISMAR. IEEE International Symposium on Mixed and Augmented Reality $15-18$. Cambridge.

www.businessway.home.pl [Accessed 17 Juny 2018] 